\title{
SEGURANÇA INTERNACIONAL E NUCLEAR NO \\ SÉCULO XXI: AMEAÇAS, DESAFIOS E \\ PERSPECTIVAS
}

Amena Martins Yassine ${ }^{1}$

\begin{abstract}
This piece of work intends to analyse the security paradigm of the $21^{\text {st }}$ century and the changes in the United States' security policy, framed to combat the proliferation of weapons of mass destruction, particularly the nuclear ones, after September $11^{\text {th }}$ 2001. For achieving such a purpose, we referred to the most relevant legislation in this area, the Non-Proliferation Treaty, the International Atomic Energy Agency Statute, the United Nations Charter, the Non-Proliferation Security Initiative Statement, and the The National Security Strategy of the United States of America. In addition, we referred to the most important scientists in the International Relations field, who are engaged in understanding the political structure of the post-Cold War era.
\end{abstract}

\section{Introdução}

Com o fim da Guerra Fria, mudanças econômicas e políticas transformaram o cenário internacional. $\mathrm{Na}$ área estratégica, por exemplo, a disputa militar entre União Soviética e Estados Unidos foi substituída pela liderança inconteste desse último. Joseph Nye compara esse novo quadro a um complexo "jogo de xadrez tridimensional", onde o poderio militar norte-americano seria predominantemente unipolar. Samuel Huntington igualmente convém na preeminência

\footnotetext{
${ }^{1}$ Amena Martins Yassine é bacharel em Relações Internacionais pelo Centro Universitário de Brasília - UniCEUB - e está se graduando em Letras (Inglês) pela Universidade de Brasília - UnB.
}

Universitas - Relações Int., Brasília, v. 2, n.2, p. 199-219, jul./dez. 2004 
estadunidense nesse aspecto. Segundo esse internacionalista, a macroestrutura de poder é hoje "uni-multipolar", sendo os Estados Unidos a superpotência militar desde o início da década passada. É justamente à análise do paradigma de segurança internacional emergente a partir da hegemonia norte-americana a que se destina o presente ensaio.

Historicamente, os esforços de controle das armas nucleares precederam aqueles das demais armas de destruição em massa (químicas e biológicas) $^{2}$. Em função do ambiente de beligerância da Segunda Guerra Mundial, a tecnologia nuclear foi prioritariamente desenvolvida com propósitos militares e mais tarde estendida a usos pacíficos - sobretudo com a assinatura do Tratado de Não-Proliferação Nuclear-TNP. O mesmo não pode ser dito para o caso de tecnologias como a química e biológica, que foram primeiramente destinadas a fins medicinais. Desse modo, procede argüir que o controle dos artefatos nucleares tem maior relevância relativa para a garantia da segurança internacional. Não se está afirmando aqui a inexpressividade das demais negociações sobre desarmamento, quer químico, quer biológico. Apenas se pretende enfatizar a prioridade histórica e hierárquica do segmento nuclear nos assuntos de não-proliferação ${ }^{3}$ para justificar a opção desse ensaio de estudar o paradigama de segurança, que emerge depois do 11 de setembro de 2001, a partir de uma análise dos desafios ao cumprimento do Tratado de NãoProliferação Nuclear-TNP. De fato, a extensão indefinida do Tratado, bem como sua abrangência em número de membros, tornou $o$ instrumento imprescindível para quaisquer investigações nessa área.

Essa pesquisa se ocupará, desse modo, da análise dos desafios ao sistema de segurança internacional e nuclear na atualidade, indicando a disseminação da energia nuclear pela Índia, Paquistão e Israel como primeiros desafios à estabilidade internacional. Outros casos de proliferação, como o iraniano e o norte-coreano também serão avaliados. Em seguida, esse trabalho se voltará para a avaliação das ações empreendidas pelos Estados Unidos, como a "Proliferation

\footnotetext{
${ }^{2}$ Op. Cit., Lamazière 1998, p. 49-54.

3 Ibidem, p. 49-54.
}

Universitas - Relações Int., Brasília, v. 2, n.2, p. 199-219, jul./dez. 2004 
Security Initiative", para previnir que grupos terroristas adquiram armas nucleares.

\section{Desafios ao Atual Sistema de Segurança Internacional e Nuclear}

O primeiro desafio ao TNP, portanto, é certamente o de evitar, senão impedir, transgressões à norma principal do tratado, qual seja a não-proliferação horizontal ${ }^{4}$. Sobre esse aspecto, cabe discutir aqui os principais casos de disseminação da energia nuclear que têm causado constrangimentos entre os Estados-parte do acordo em referência. Fazse oportuno lembrar, portanto, que a Índia, Paquistão e Israel, embora detenham a arma nuclear, não se incorporam ao TNP. Um elemento jurídico aprofunda essa tensão: a inviabilidade de se reconhecer aqueles Estados como potências nucleares caso os mesmos quisessem vir a ser parte do tratado. O artigo IX, parágrafo 3, do acordo dispõe que "um Estado nuclearmente armado é aquele que tiver fabricado ou explodido uma arma nuclear ou outro artefato explosivo nuclear antes de 1o. de janeiro de 1967. "Sabendo que a Índia e Paquistão realizaram seus testes nucleares no final da década de 1990, esses, legalmente, não poderiam se juntar ao clube dos países nucleares. Desse quadro mundial certamente surge uma crise de credibilidade no TNP. Conferir status jurídico aos dois países abriria precedente para novos candidatos buscarem o desenvolvimento da arma nuclear. Não obstante a aprovação pelo Conselho de Segurança da resolução 1172 (1998) que lhes nega o reconhecimento de jure da nova postura, Índia e Paquistão ainda vivem à margem do TNP. Se existir uma disposição das potências nucleares no sentido de acomodar a situação indiana, a

\footnotetext{
${ }^{4}$ O termo "proliferação vertical" inclui limitação do crescimento numérico e qualitativo das armas nucleares, enquanto que "proliferação horizontal" diz respeito apenas a limitação do acesso a tecnologias nucleares por outros Estados. Cf. SCHMALBERGER, Thomas e TULLIU, Steve (org.). Coming to Terms with Security: A Lexicon for Arms Control, Disarmament and Confidence-Building. Switzerland: United Nations Institute for Disarmament Research, 2003.

${ }^{5}$ Cf. Tratado de Não-Proliferação Nuclear, artigo IX, parágrafo 3.
}

Universitas - Relações Int., Brasília, v. 2, n.2, p. 199-219, jul./dez. 2004 
possibilidade de que outros Estados reconsiderem as vantagens estratégicas de integrar o instrumento em questão não é desprezada. $\mathrm{O}$ mesmo raciocínio se estende a Israel, que igualmente possui a bomba, mas não é parte do acordo.

Outra dificuldade que poderia enfrentar o tratado diz respeito a novos casos de proliferação horizontal que podem se configurar como uma ameaça aos interesses dos países comprometidos com o controle de energia nuclear e temerosos da própria segurança nacional. Assim, cumpre avaliar as investidas iranianas e norte-coreanas no desenvolvimento de um arsenal nuclear. A principal diferença entre Irã e os Estados nucleares de facto referidos anteriormente é que Teerã se compromenteu com o TNP. Portanto, a estratégia mais eficiente para lidar com os iranianos seria a aplicação dos dispositivos de nãoproliferação do TNP, o que fortaleceria o tratado. Instrumentos de dissuasão, legitimados pela comunidade internacional, como os aplicados a Bagdá, também seriam úteis e contribuiriam para desencorajar novas tentativas de proliferação horizontal. Como se tem ciência, o Iraque ficou sujeito, por meio da resolução 687 (1991) do Conselho de Segurança, a verificações e monitoramentos consideravelmente rígidos $^{6}$, que permitiram aos inspetores da Agência Internacional de Energia Atômica-AIEA o desmantelamento da capacidade nuclear do país ${ }^{7}$.

Infrações cometidas contra o TNP não podem ser tratadas, contudo, com medidas que comprometam o princípio, contido no

${ }^{6}$ Sobre esse assunto ver op. Cit. LAMAZIÈRE, Georges. Ordem, Hegemonia e Transgressão: a Resolução 687 (1991) do Conselho de Segurançã das Nações Unidas, a Comissão Especial das Nações Unidas e o regime internacional de não proliferação de armas de destruição em massa. Brasília: Instituto Rio Branco; Fundação Alexandre Gusmão; Centro de Estudos Estratégicos, 1998.

${ }^{7}$ Ressalva-se que, não obstante as recentes suspeitas estadunidenses sobre as atividades nucleares do Iraque, a declaração do Diretor-Geral da AIEA, Mohamed EIBaradei, a 27 de janeiro de 2003, , em resposta à resolução 1441 (2002), que autoriza novas inspeções no Iraque, conclui que: "we have to date found no evidence that Iraq has revived its nuclear weapons programme since the elimination of the programme in the 1990s." Cf. <http://www.un.org/News/dh/iraq/elbaradei27jan03.htm> (Acesso: 20 maio de 2004)

Universitas - Relações Int., Brasília, v. 2, n.2, p. 199-219, jul./dez. 2004 
preâmbulo do acordo, segundo o qual "os benefícios das aplicações pacíficas da tecnologia nuclear (...) devem ser postos, para fins pacíficos, à disposição de todas as Partes do Tratado, sejam elas Estados nuclearmente armados ou não. ${ }^{81}$ Tampouco devem ferir o Artigo IV, parágrafos 1 e 2, que versa sobre o "direito inalienável de todas as Partes do Tratado de desenvolverem a pesquisa, a produção e a utilização da energia nuclear para fins pacíficos ${ }^{91}$ e sobre o "intercâmbio possível de equipamento, materiais e informação científica e tecnológica sobre a utilização pacífica da energia nuclear. $^{10}$ " $\mathrm{O}$ desrespeito a tais regras apenas desacreditaria o TNP, uma vez que seus Estados-parte reconsiderariam os benefícios advindos da imposição desse arcabouço normativo. Cabe esclarecer que as adesões ao TNP foram, em grande medida, motivadas pelas campanhas americana, britânica e russa, cujos propósitos eram sublinhar como vantagens do tratado o fato de que ele "... would increase the security of both nuclear and non-nuclear-weapon States, would enable all nations, particularly the developing nations, to share in the benefits of peaceful applications of nuclear energy, and would facilitate the cessation of the nuclear arms race. ${ }^{11 "}$ (grifo nosso) Nesse sentido, ações que desconsiderem os preceitos acima representarão, antes, um terceiro desafio ao sistema de segurança nuclear. Alternativas que surgem para dar fim a esse problema, como a "Proliferation Security Initiative", que será discutida em seção posterior, ilustram que os EUA estão desconsiderando tal risco.

O caso norte-coreano é particularmente emblemático, uma vez que este país tornou-se o primeiro a denunciar o TNP, a 10 de abril de 2003. Na verdade, a intenção de Pyongyan de se retirar do tratado é conhecida desde 1993, quando entendimentos com os Estados Unidos levaram Kim Jong Il a reconsiderar a decisão de sair. Aparentemente, o intento de deixar o TNP era o de evitar inspeções pela AIEA que possivelmente iriam revelar desvios de material nuclear. De fato, o Artigo $\mathrm{X}$ do tratado é bastante permissivo ao prescrever que cada

\footnotetext{
${ }^{8}$ Cf. Tratado de Não-Proliferação Nuclear.

${ }^{9}$ Ibidem, Artigo IV.

${ }^{10}$ Ibidem, Artigo IV, parágrafos 1 e 2.

${ }^{11}$ Op. Cit., The United Nations and Disarmament: 1945-1970, p.293.
}

Universitas - Relações Int., Brasília, v. 2, n.2, p. 199-219, jul./dez. 2004 
Estado tem o direito de denunciar o Tratado se decidir que "acontecimentos extraordinários" põem em risco os interesses supremos do país. Se ao integrar o TNP os membros do acordo passam a dispôr de facilidades no acesso a materiais, tecnologia, equipamentos e informações relativos ao uso da energia nuclear, o processo de retirada do acordo deveria ser mais rígido e menos subjetivo. De todo modo, essa nova realidade internacional insere mais uma preocupação quanto à proliferação horizontal, sobretudo porque há suspeitas, como revela a revista The Economist ${ }^{12}$, de que a Coréia do Norte possa ter produzido cerca de oito bombas. Uma vez que os termos do TNP não mais vinculam compromissos por parte do país asiático, a solução para a questão deveria considerar primeiramente o retorno do país ao Tratado.

Apesar da centralidade da não-proliferação horizontal, há que se dizer que, findada a Guerra Fria e o "equilíbrio do terror", novas expectativas surgem com respeito ao controle de armamentos. De fato, a ausência de rivalidades no cenário internacional, ensejou novas negociações sobre o desarmamento, incluindo o vertical, coordenado pela potência reinante, cujos ápices são os entendimentos sobre o Tratado para a Proibição Completa dos Testes Nucleares-CTBT e os "13 Passos para o Desarmamento". Cabe argumentar, portanto, que a atual posição norte-americana, em resposta ao atentados de 11 de setembro, de rechaçar quaisquer compromissos sobre essa matéria, representa igualmente um desafio à estabilidade do sistema de segurança nuclear. $\mathrm{O}$ quadro exposto se agrava quando se nota a possibilidade de uma retomadada da corrida armamentista nuclear.

\section{O recrudescimento da proliferação vertical e da corrida armamentista nuclear}

De fato, o fim das tensões americano-soviéticas desanuviou o clima de corrida armamentista, tornando anacrônica a percepção de que reter e aperfeiçoar armas nucleares era fundamental para $o$

\footnotetext{
${ }^{12}$ Cf. The Economist. "Resign, Rumsfeld". Vol. 371. No. 8374. London, 8-14 de maio de 2004, p.32.
}

Universitas - Relações Int., Brasília, v. 2, n.2, p. 199-219, jul./dez. 2004 
equilíbrio entre as duas potências e, por conseguinte, para a estabilidade mundial. À medida que o mundo pós-Guerra Fria afastou a ênfase na necessidade de armas nucleares e vetores de lançamento cada vez mais sofisticados, criou também ambiente apropriado para se barganhar a redução do estoque dessas armas, como demonstraram os resultados do processo de exame do TNP durante a Conferência de Revisão do acordo em 2000.

A política de segurança norte-americana, transmutada em função dos atentados de 11 de setembro de 2001, começa, entretanto, a destoar do consenso de segurança prevalecente na década de 1990. Dois elementos ilustram a mudança de postura estadunidense: a doutrina de Segurança Estratégica dos Estados Unidos, e o desenvolvimento de novas capacidades nucleares. O aspecto mais significativo da estratégia em questão é certamente o fato de que ela considera a possibilidade do "uso em primeira instância" das armas nucleares, inovando, assim, o conceito de deterrence. Quanto às pesquisas nucleares, o mais importante é que elas lançam as bases para uma nova corrida armamentista. Sem dúvida, o quadro de rivalidades que caracteriza o início deste século pode ressuscitar a ênfase nas armas nucleares como mecanismo de segurança das nações.

Sobre esse aspecto, Samuel Huntington faz uma observação interessante:

During the Cold War the United States and the Soviet Union engaged in a classic arms race, developing more and more technologically sophisticated nuclear weapons and delivery vehicles for them. It was a case of buildup versus buildup. In the post-Cold War world the central arms competition is of a different sort. The West's antagonists are attempting to acquire weapons of mass destruction and the West is attempting to prevent them from doing so. It is

Universitas - Relações Int., Brasília, v. 2, n.2, p. 199-219, jul./dez. 2004 
not a case of buildup versus buildup but rather of buildup versus hold-down. ${ }^{13}$ (grifo nosso)

Nesse sentido, Huntington parece assumir que a postura estadunidense é de manter o acúmulo ("buildup") de armas nucleares e deter ("hold-down") a disseminação das mesmas. Na prática, é possível observar que a Administração Bush efetivamente compartilha a idéia de seguir com o processo de desenvolvimento de novas capacidades nucleares. Em 2003, o Congresso americano rejeitou a emenda "SprattFurse" de 1993 que bania pesquisa e desenvolvimento de "low-yield nuclear weapons" e aprovou verba de US\$ 6 milhões para o início dos trabalhos nessa área ${ }^{14}$. A Força Aérea dos EUA também está discutindo o desenvolvimento de armas nucleares ("Robust Nuclear Earth Penetrator"-RNEP) com capacidade de atacar alvos subterrâneos profundos, tornando o uso desses arsenais mais factível. Além disso, os EUA continuam a recusar o Tratado para a Proibição Completa dos Testes Nucleares. Por ocasião da Assembléia-Geral das Nações Unidas, em 2003, o Embaixador Americano Stephen Rademaker asseverou: "The U.S. does not support the Comprehensive Nuclear Test-Ban Treaty and will not become part to it. ${ }^{15}$ " $\mathrm{O}$ aperfeiçoamento das armas nucleares sugere que a política de defesa americana não estaria inclinada a implementar ações com vistas à eliminação de tais dispositivos.

São igualmente notórias as iniciativas de "hold-down" adversários que confrontam os EUA no pós-Guerra Fria. Durante a reunião do G8, a 31 de março de 2003, por exemplo, o Presidente Bush anunciou o projeto "Proliferation Security Initiative", cujo objetivo é outorgar a onze países (EUA, Austrália, França, Alemanha, Itália, Japão, Países Baixos, Polônia, Portugal, Espanha e Reino Unido) o direito de interceptar carregamentos marítimos, terrestres e aéreos

${ }^{13}$ Cf. HUNTINGTON, Samuel. The Clash of Civilizations and the Remarking of World Order. (Simon \& Schuster: London, 1996), p. 190.

${ }^{14} \mathrm{Cf}$. NGO Shadow Report on Nuclear Disarmament. Accountability is Democracy, Transparency is Security. (WILPF: New York, april 2004), p. 6.

${ }^{15}$ Ibidem, p. 1.

Universitas - Relações Int., Brasília, v. 2, n.2, p. 199-219, jul./dez. 2004 
suspeitos de traficar armas de destruição em massa, tecnologias e equipamentos relacionados a mísseis, além de outros equipamentos ${ }^{16}$.

Huntington reconhece que "The West promotes nonproliferation as reflecting the interests of all nations in international order and stability. Other nations, however, see nonproliferation as serving the interests of Western hegemony. ${ }^{17}$ " Para o cientista, a globalização econômica e o desenvolvimento social propiciaram a difusão de capacidades militares em sociedades nãoOcidentais, sobremaneira por causa das transferências de tecnologias militares da China e Rússia para países como o Irã, Paquistão, Coréia do Norte e Iraque. Esse processo, prevê Huntington, irá persistir e se agravar nos primeiros anos do Séc. XXI. Segundo Huntington, a força militar Ocidental é imbatível e, por conseguinte, as sociedades nãoOcidentais procurarão mecanismos para contrabalançar o poderio do inimigo por meio do acesso a armas de destruição em massa e seus vetores de lançamento. Oriente, comenta o estudiosio, crê que "if you have nuclear weapons, the United States won't fight you ${ }^{18 " . ~ A s ~ a r m a s ~}$ nucleares servem, portanto, como elemento equilibrador da estrutura de poder vigente. Aliado a essa tendência, o terrorismo certamente figura como importante desafio para o sistema de segurança. Nas palavras de Huntington,

Terrorism historically is the weapon of the weak, that is, of those who do not possess conventional military power. Since World War II, nuclear weapons have also been the weapon by which the weak compensate for conventional inferiority. In the past, terrorists could do only limited violence, killing a few people here or destroying a facility there. Massive military forces were required to do massive violence. At

\footnotetext{
16 Cf. sítio do Departamento de Estado Norte-Americano: $<$ http://www.state.gov/t/np/rls/fs/23764pf.htm> (acesso: 23 de outubro de 2003)

${ }^{17}$ Op. Cit., Huntington 1996, p. 1991.

${ }^{18}$ Op. Cit., Huntington 1996, p.187.
}

Universitas - Relações Int., Brasília, v. 2, n.2, p. 199-219, jul./dez. 2004 
some point, however, a few terrorists will be able to produce massive violence and massive destruction. Separately, terrorism and nuclear weapons are the weapons of the non-Western weak. If and when they are combined, the nonWestern weak will be strong. ${ }^{19}$

Os esforços para produção de armas nucleares no mundo pósGuerra Fria estão concentrados nos Estados islâmicos e confucionistas, e são resquícios de anos de cooperação entre os países comunistas e muçulmanos. De 1980 a 1991, faz lembrar o pesquisador, Irã e Paquistão eram os principais recipiendários das armas e tecnologias militares da China. O Irã também recebeu auxílio técnico da Coréia do Norte e Paquistão, igualmente apoiados pela China. O resultado dessa disseminação levou a questão da não-proliferação ao centro da agenda de segurança dos EUA. Diferentemente dos anos de Guerra Fria, entretanto, comenta Huntington,

The outcome of a race between buildup and hold-down is more predictable. The hold-down efforts of the West may slow the weapons buildup of other societies, but they will not stop it. The economic and social development of non-Western societies, the commercial incentives for all societies Western and nonWestern to make money through the sale of weapons, technology, and expertise, and the political motives of core states and regional powers to protect their local hegemonies, all work to subvert Western hold-down efforts. ${ }^{20}$

A explicação para o fracasso das políticas de não-proliferação segundo Huntington é que as potências regionais entendem que as armas nucleares "enable those states to establish their dominance over

\footnotetext{
${ }^{19}$ Op. Cit., Huntington 1996, p.188.

${ }^{20}$ Ibidem, p.191.
}

Universitas - Relações Int., Brasília, v. 2, n.2, p. 199-219, jul./dez. 2004 
other states in their civilization and region, and, second, provide them with the means to deter intervention in their civilization and region by the United States or other external powers. ${ }^{21 "}$ A percepção desse pesquisador lembra, em muitos aspectos, as concepções da Escola Inglesa das Relações Internacionais, cujos maiores expoentes são Martin Wight e Hedley Bull. Para esses autores, os Estados existem em uma condição de anarquia e o sistema internacional é uma "arena na qual os homens de estado perseguem seus interesses e periodicamente chegam a conflitos que podem ameaçar a sobrevivência de alguns. ${ }^{22 "}$ Nesse sentido, para garantir a posição de dominância, a potência mais forte será obrigada a assumir a liderança das inovações no campo militar e buscar mecanismos para congelar o status quo.

Esse ensaio se distancia da visão da escola inglesa. Contudo, a posição aqui defendida não é menos realista que a de Huntington, tampouco mais grociana, uma vez que se reconhece o caráter peculiar da área de segurança, ao qual se referem Jervis e Lamazière. O que se propõe aqui nada mais é que o fortalecimento do instrumento de segurança surgido para acomodar a estrutura de poder das potências nucleares. 188 países estão organizados sob um arcabouço jurídico de grande legitimidade, desse modo, os esforços dos Estados Unidos e de outras potências Ocidentais para combater as ameaças do Séc. XXI não devem configurar per se desafios à ordem consolidada por esses. Do contrário, seria forçoso acreditar que, nas palavras de Huntington, "The efforts by the United States and other Western countries to prevent the proliferation of 'equalizer' weapons of mass destruction (...) are likely to continue to meet with limited success. ${ }^{23 "}$

A se medir pela proposta do Secretário para Controle de Armas e Segurança Internacional dos Estados Unidos, John Bolton, conhecida como "Proliferation Security Initiative", os resultados prenunciados por Huntington, e reproduzidos a seguir, podem efetivamente ser levados a cabo:

\footnotetext{
${ }^{21}$ Ibidem, p.186.

22 Op. Cit., Wight, prefácio, p. XV.

${ }^{23}$ Op. Cit., Huntington 1996, 192.
}

Universitas - Relações Int., Brasília, v. 2, n.2, p. 199-219, jul./dez. 2004 
In 1993 the primary goals of the West, as defined in American policy, shifted from nonproliferation to counterproliferation. This change was a realistic recognition of the extent to which some nuclear proliferation could not be avoided. In due course, U.S. policy will shift from countering proliferation to accommodating proliferation [caso da Índia, Paquistão e Israel?] and, if the government can escape from its Cold War mind-set, to how promoting proliferation can serve U.S. and Western interests. As of 1995, however, the United States and the West remained committed to a hold-down policy which, in the end, is bound to fail. The proliferation of nuclear and other weapons of mass destruction is a central phenomenon of the slow but ineluctable diffusion of power in a multicivilizational world. ${ }^{24}$ (grifo nosso)

Dado o caráter permanente e a amplitude do TNP, medidas que se centrem apenas na proliferação horizontal e que desconsiderem os avanços advindos da Conferência de Revisão de 2000 poderão estar fadadas ao fracasso. Se alternativas políticas para o combate ao terrorismo ameaçarem a operacionalização do TNP, restará apenas concluir que esse dispositivo já não mais é capaz de responder aos propósitos das potências nucleares, em especial aos dos Estados Unidos.

O próprio Huntington lembra que durante a conferência de revisão do TNP em 1995, os Estados Unidos queriam a extensão indefinida do tratado, mas vários países se manifestaram contra a proposta porque iniciativas mais drásticas de redução das armas nucleares não estavam sendo cogitadas. O Egito, exemplifica o pesquisador, exigiu a entrada de Israel no TNP como condição da aceitação da extensão. "In the end, the United States won an overwhelming consensus on indefinite extension through a highly

\footnotetext{
${ }^{24}$ Op. Cit., Huntington 1996, p. 192.
}

Universitas - Relações Int., Brasília, v. 2, n.2, p. 199-219, jul./dez. 2004 
successful strategy of arm twisting, bribes, and threats. ${ }^{25 "}$ Egito $\mathrm{e}$ México, conta Huntington, não puderam sustentar suas posições devido à dependência aos EUA. De fato, a segurança coletiva não pode estar sustentavelmente assegurada por meio de soluções como as encontradas para tal encontro. Não soubessem os Estados Unidos disso, o TNP não teria sido forjado entre os demais países.

\section{Proliferation Security Initiative: Instância de Otimização dos Instrumentos de Segurança Nuclear?}

Para conter o problema da proliferação, o Presidente George Bush lançou, a 31 de maio de 2003, por ocasião da reunião do G8, em Cracóvia, Polônia, a "Proliferation Security Initiative-PSI". A PSI não pretende ser um tratado, tampouco uma organização internacional, mas, antes, um conjunto de ações coordenadas (uma "Força Tarefa"), envolvendo Estados "comprometidos com a não-proliferação", com a finalidade de interceptar embarcações "suspeitas" de transportarem armas de destruição de massa, vetores de lançamento e/ou qualquer material relacionado, seja por via aérea, seja marítima ou terrestre ${ }^{26}$.

Segundo informa o Departamento de Estado Americano, as ações desempenhadas no âmbito da PSI serão consistentes com as "existing national legal authorities and international law and frameworks. ${ }^{27 "}$ Nesse sentido, as parcerias envolvidas em tal trabalho iriam apenas aplicar "their national capabilities to develop a broad range of legal, diplomatic, economic, military, and other tools to interdict shipments of such items. ${ }^{28 "}$ (grifo nosso)

Fazem parte da Força Tarefa os Estados Unidos, Austrália, França, Alemanha, Itália, Japão, Países Baixos, Polônia, Portugal, Espanha e Reino Unido, que se reuniram pela primeira vez em Madri, a 15 de junho de 2003. Oficialmente, a eleição dos onze países se deu porque "[they] all have demonstrated strong support for

${ }^{25}$ Ibidem, p. 192.

Cf. Sítio do Departamento de Estado Americano: $<$ http://www.state.gov/t/np/rls/fs/32725.htm> (Acesso: 1 de junho 2004)

${ }^{27}$ Ibidem.

${ }^{28}$ Ibidem.

Universitas - Relações Int., Brasília, v. 2, n.2, p. 199-219, jul./dez. 2004 
nonproliferation; have been involved in efforts to prevent proliferation, including active interdiction efforts; and are located in geographically important locations in relation to proliferation pathways. ${ }^{29 "}$ Além disso, o número restrito de participantes, informa o Departamento de Estado, tem se mostrado "eficiente" e "produtivo" nessa fase inicial do programa. Candidatos desejosos de integrar a operação devem, em primeiro, demonstrar apoio oficial à carta de princípios ("Statement of Interdiction Principles") pelos onze definida a 3-4 de setembro de 2003, em Paris.

O mais significativo para os propósitos desse artigo é saber como a PSI irá interagir com as normas de não-proliferação já existentes, sobremaneira com o Tratado de Não-Proliferação de Armas Nucleares. Para tanto, faz-se oportuno analisar os princípios que orientam a PSI, bem como seu objetivo original, que se fazem reproduzidos abaixo:

PSI participants are committed to the following interdiction principles to establish a more coordinated and effective basis through which to impede and stop shipments of WMD, delivery systems, and related materials flowing to and from states and non-state actors of proliferation concern, consistent with national legal authorities and relevant international law and frameworks, including the UN Security Council. ${ }^{30}$ (grifo nosso)

Uma avaliação geral sobre os aspectos jurídicos do objetivo da PSI é fundamental, sobretudo naquilo que diz respeito ao direito do mar, regulado pela Convenção das Nações Unidas para o Direito do Mar, de 1982. A regra geral sobre o trânsito em alto mar, Artigo 86 da Convenção, diz que "o alto mar está aberto a todos os Estados, quer costeiros quer sem litoral. " Isso implica, obviamente, limites à ação

${ }^{29}$ Ibidem.

${ }^{30}$ Op. Cit., Sítio do Departamento de Estado Americano, acesso: 2 de junho de 2004.

Universitas - Relações Int., Brasília, v. 2, n.2, p. 199-219, jul./dez. 2004 
soberana em alto mar. De fato, o Artigo 89 do mesmo tratado dispõe que "nenhum Estado pode legitimamente pretender submeter qualquer parte do alto mar à sua soberania." Entretanto, a reivindicação da soberania sobre o alto mar não está proibida. Basta lembrar que é possível abordar navios "piratas" ${ }^{31}$ ou com cargas consideradas ilegais (escravos, substâncias psicotrópicas, estupefacientes etc) pela legislação internacional ${ }^{32}$. Desse modo, a interdição de navios suspeitos de transportarem armas de destruição em massa não seria ilegal, sobretudo se for considerado o fato de que a proliferação desses dispositivos está proibida por instrumentos internacionais. Além disso, a interpretação do artigo 88 da Convenção, que determina o uso do mar para fins pacíficos, poderia eventualmente garantir a legalidade de tais práticas. Resta mencionar que "The United Nations Security Council just recently voted [a 28 de abril de 2004] on the Proliferation Security Initiative (PSI) resolution. ${ }^{33 "}$

Sendo assim, torna-se mais apropriado uma discussão em torno da legitimidade da Iniciativa, bem como de suas implicações para o tratamento das questões de segurança internacional. Os princípios da Declaração que interessam a essa análise são os dois primeiros, porque eles inserem uma preocupação política com respeito à definição de "states or non-states actors of proliferation concern" e aos critérios de identificação de tais "suspeitos". Segundo o documento, o termo em referência compreende

countries or entities that the PSI participants involved establish should be subject to interdiction activities because they are engaged in proliferation through: (1) efforts to develop or acquire chemical, biological, or nuclear weapons and associated delivery systems; or (2) transfers

\footnotetext{
${ }^{31}$ Para definição de "pirataria" ver Convenção das Nações Unidas para o Direito do Mar, de 1982, artigo 101.

${ }^{32}$ Cf. Convenção das Nações Unidas para o Direito do Mar, de 1982, artigos 99, 100 e 108.

${ }^{33}$ Cf. Sítio da Embaixada dos EUA na Austrália:

$<$ http://usembassy-australia.state.gov/hyper/2004/0428/epf303.htm> (acesso: 1 de junho de 2004).
}

Universitas - Relações Int., Brasília, v. 2, n.2, p. 199-219, jul./dez. 2004 
(either selling, receiving, or facilitating) of WMD, their delivery systems, or related materials. $^{34}$

O grau de envolvimento em atividades de proliferação será constatado, como determina o princípio dois da Carta, por meio de "relevant information". Segundo o governo americano, "the United States only pursues interdiction efforts when we believe there is a solid case for doing so. ${ }^{35 "} \mathrm{Na}$ verdade, a recente campanha dos EUA contra o Iraque sob o argumento de que esse país dispunha de capacidade de armas de destruição em massa e a posterior constatação da inexistência dessas mesmas impingiram desconfiança sobre a isenção estadunidense no trato dos assuntos de segurança. Acredita-se, portanto, que os princípios acima referidos serão um ponto de dissensão entre os demais membros da comunidade internacional, que temerão tratamentos diferenciados e discriminatórios.

A Iniciativa, garante o Departamento de Estado, "does not replace other nonproliferation mechanisms, but reinforces and complements them. ${ }^{36 "}$ Um olhar cauteloso sobre o Artigo III do TNP sugere, no entanto, que a PSI, no mínimo deslegitima a Agência Internacional de Energia Atômica em seu papel de reguladora dos transportes de energia nuclear:

Cada Estado não-nuclearmente armado, Parte deste Tratado, compromete-se a aceitar salvaguardas - conforme estabelecidas em um acordo a ser negociado e celebrado com a Agência Internacional de Energia Atômica, de acordo com o Estatuto da Agência Internacional de Energia Atômica e com o sistema de

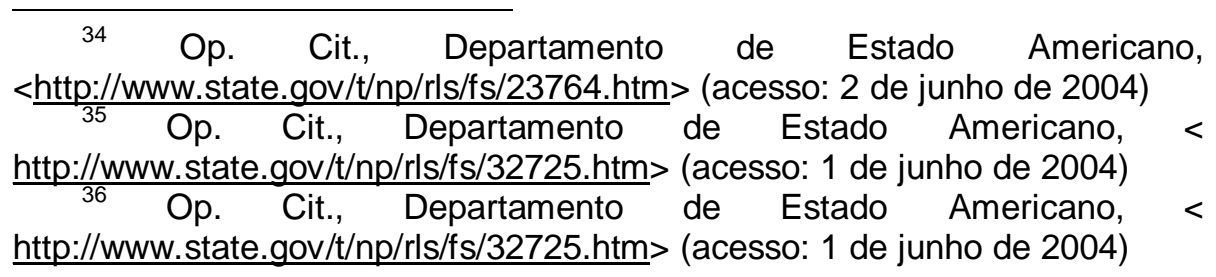

Universitas - Relações Int., Brasília, v. 2, n.2, p. 199-219, jul./dez. 2004 
salvaguardas da Agência - com a finalidade exclusiva de verificação do cumprimento das obrigações assumidas sob o presente Tratado, e com vistas a impedir que a energia nuclear destinada a fins pacíficos venha a ser desviada para armas nucleares ou outros artefatos explosivos nucleares. ${ }^{37}$

Dada a possibilidade de tratamento discriminatório, cabe argumentar além: o Artigo VI, parágrafo 2 do TNP que determina que todas as Partes do tratado se comprometerá "a facilitar o mais amplo intercâmbio possível de equipamento, materiais e informações científicas e tecnológicas sobre a utilização pacífica da energia nuclear e dele têm o direito de participar ${ }^{38 " ~ f i c a ~ i g u a l m e n t e ~}$ comprometido. Resta dizer ainda que o modelo em questão, que na falta de melhor definição poderia ser denominado de "multilateralismo seletivo", desvaloriza os mecanismos multilaterais de tratamento dos assuntos de não-proliferação e desarmamento, tornando mais espinhosos quaisquer avanços sobre este último aspecto. À medida que o trato dessas questões passa a ser coordenado de modo seletivo, o poder de barganha dos "non-like minded states" fica reduzido, como também o fica quaisquer avanços na área de não-proliferação vertical.

\section{Considerações Finais}

A segurança internacional funciona segundo uma lógica de poder. Durante os anos da Guerra Fria essa lógica era orientada pela estrutura de poder bipolar, centrada no equilíbrio entre o poderio militar americano e soviético. Com o declínio da União Soviética, a segurança internacional está assegurada pela hegemonia norteamericana. Todo arcabouço normativo existente na área de segurança internacional, sobretudo no campo nuclear, que advém da relação de poder entre Estados Unidos e União Soviética, parece não mais servir para os propósitos da única potência reinante. Durante a década de

\footnotetext{
${ }^{37}$ Op. Cit., Tratado de Não-Proliferação Nuclear, artigo III.

${ }^{38}$ Ibidem, artigo IV.
}

Universitas - Relações Int., Brasília, v. 2, n.2, p. 199-219, jul./dez. 2004 
1990, a potência hegemônica apenas assegurou o consenso em torno das normas já estabelecidas. Desde 11 de setembro de 2001, entretanto, iniciativas como a PSI procuram ajustar o arcabouço normativo herdado à nova estrutura de poder que se arma, aquela onde, no dizer do Presidente Bush, "small groups of fanatics, or failing states, could gain the power to threaten great nations, threaten the world peace. ${ }^{39 "}$. (grifo nosso) A sustentabilidade da segurança internacional e nuclear dependerá, portanto, para aplicar as teses de Gilpin na área de segurança, da capacidade dos Estados Unidos de conseguirem consenso sobre sua nova estratégia de segurança e sobre as iniciativas que coordenam com a finalidade de conter a proliferação horizontal. Do contrário, o fracasso operacional da Iniciativa atestará se as hipóteses huntingtonianas se confirmam.

Se a luta contra o terrorismo for travada de maneira a deslegitimar o TNP, restará ao presente trabalho a conclusão de que a paz e segurança internacionais dependerão da capacidade de os Estados Unidos conseguirem consenso sobre a necessidade de maior rigidez para o controle horizontal de armamentos e maior flexibilidade para o seu avanço nas pesquisas para o aperfeiçoamento de suas armas nucleares.

\section{Referências bibliográficas}

AMADO, Rodrigo (org.). Araújo Castro. Brasília: UnB, 1982.

ARON, Raymond. Paz e guerra entre as nações. Brasília: Editora Universidade de Brasília, 2002.

BOBBIO, Noberto et al. Dicionário de Política. Brasília: Unb, 1995.

BULL, Hedley. Sociedade Anarquica. Brasília: UnB, Instituto de Pesquisa de Relações Internacionais; São Paulo: Imprensa Oficial do Estado de São Paulo, 2002.

BROWN, Chris. Understanding International Relations. New York: PALGRAVE, 2001.

$\begin{array}{cccccc}39 & \text { Cf. } & \text { Sítio da } & \text { National } & \text { Defense University: } \\ \text { http://ndu.edu/info/whatsnew/PresBush-NDU.cfm, } 09 \text { de março de } 2003 .\end{array}$

Universitas - Relações Int., Brasília, v. 2, n.2, p. 199-219, jul./dez. 2004 
COX, Robert W. Approaches to World Order. Cambridge: Cambridge University Press, 1996.

DOUGHERTY, James E. e PFALTZGRAFF Jr., Robert L.Contending theories of International Relations: a comprehensive survey. 5. Ed. New York: Longman, 2001.

FERNANDES, Luis e REBELO, Aldo (org.) Seminário de Política de Defesa para o Século XXI. Brasília: Câmara dos Deputados, Coordenação de Publicações, 2003.

FONSECA Jr., Gelson. A Legitimidade e outras Questões Internacionais: poder e ética entre as nações. São Paulo: Paz e Terra, 1998.

Fundação Alexandre Gusmão. La Palabra del Brasil en las Naciones Unidas, 1946-1995. Brasília: FUNAG, 1995.

GILPIN, Robert. The Political Economy of International Relations. New Jersey: Princeston University Press, 1987.

HOBSBAWM, Eric. Era dos Extremos: o breve século XX:1914-1991. São Paulo: Companhia das Letras, 1995.

HUNTINGTON, Samuel. The Clash of Civilizations and the Remarking of World Order. London: Simon \& Schuster,1996. KISSINGER, Henry. Diplomacy. New York: Touchstone, 1994. Does America need a Foreign Policy? Toward a diplomacy for the $21^{\text {st }}$ century. New York: Simon \& Schuster, 2001.

KRASNER, Stephen. International Regimes. Ithaca: Cornell University Press, 1983.

LAMAZIÈRE, Georges. Ordem, Hegemonia e Transgressão: a Resolução 687 (1991) do Conselho de Segurançã das Nações Unidas, a Comissão Especial das Nações Unidas e o regime internacional de não proliferação de armas de destruição em massa. Brasília: Instituto Rio Branco; Fundação Alexandre Gusmão; Centro de Estudos Estratégicos, 1998.

MAZZUOLI, Valério de Oliveira (org.). Coletânea de Direito Internacional. São Paulo: Revista dos Tribunais, 2004.

NGO Shadow Report on Nuclear Disarmament. Accountability is Democracy, Transparency is Security. New York: WILPF, april 2004.

Universitas - Relações Int., Brasília, v. 2, n.2, p. 199-219, jul./dez. 2004 
NYE Jr., Joseph S. O Paradoxo do Poder Americano: por que a única superpotência do mundo não pode prosseguir isolada. São Paulo: UNESP, 2002.

PATRIOTA, Antonio de Aguiar. O Conselho de Segurança após a Guerra do Golfo: a Articulação de um Novo Paradigma de Segurança Coletiva. Brasília: IRBr, FUNAG; Centro de Estidos Estratégicos, 1998.

ROCHA, Antonio Jorge Ramalho da. Relações Internacionais: teorias e agendas. Brasília: IBRI, 2002.

Organização das Nações Unidas. The United Nations and Disarmament: 1945-1970. New York: UN, 1970.

SARAIVA, José Flávio Sombra (org.). Relações Internacionais: dois séculos de História: entre a ordem bipolar e o policentrismo (de 1947 a nossos dias). Volume I. Brasília: IPRI, 2001.

Relações Internacionais: dois séculos de história: entre a ordem bipolar e o policentrismo (de 1947 a nossos dias). Volume II. Brasília: IBRI, 2001.

SCHMALBERGER, Thomas e TULLIU, Steve (org.). Coming to Terms with Security: A Lexicon for Arms Control, Disarmament and Confidence-Building. Switzerland: United Nations Institute for Disarmament Research, 2003.

TEIXEIRA, Pascal. The Security Council at the Dawn of the TwentyFirst Century: to What Extent is it Willing and Able to Maintain International Peace and Security? Geneva: UNIDIR; IFRI; UN, 2003. WEIGALL, David. International Relations: a concise companion. Oxford: Oxford University Press, 2002.

WIGHT, Martin. A Política do Poder. Brasília: UnB, Instituto de Pesquisa de Relações Internacionais; São Paulo: Imprensa Oficial do Estado de São Paulo, 2002.

GADDIS, John Lewis. "A Grand Strategy". In Foreign Policy. No. 133. Ano: novembro/dezembro de 2002.

KAYSEN, Carl; McNAMARA, Robert e RATHJENS, George. "Nuclear Weapons After the Cold War". In Foreign Affairs. Vol. 70. No. 4. New York: Council on Foreign Relations, 1990/91.

Política Externa. "EUA e a Ordem Mundial: uma Nova Doutrina?". Vol. 12. No. 1. Ano: junho, julho, agosto de 2003.

Universitas - Relações Int., Brasília, v. 2, n.2, p. 199-219, jul./dez. 2004 
The Economist. "Resign, Rumsfeld". Vol. 371. No. 8374. London, 814 de maio de 2004.

VEGA, José Fernandez. "As melhores armas nas mãos do melhor sistema". In Política Externa. Vol. 12. No. 4. Ano: março, abril, maio 2004.

WEINGERG, Steven. "What price glory?" In The New York Review of Books. Vol. L. No. 17. Ano: 6 de novembro de 2003

Carta das Nações Unidas

Convenção das Nações Unidas para o Direito do Mar, de 1982

Tratado sobre a Não-Proliferação de Armas Nucleares

Estatuto da Agência Internacional de Energia Atômica

International Atomic Energy Agency: http://iaea.org

Casa Branca: http://whitehouse.gov

Central Intelligence Agency: http://www.cia.gov

Centre for Strategic and International Studies: http://www.csis.org

Nações Unidas: http://www.un.org

National Defense University: http://ndu.edu/info/whatsnew/PresBush-

NDU.cfm

US State Department: http://www.state.gov

Reaching Critical Will: Women's International League for Peace and Freedom: http://reachingcriticalwill.org

Universitas - Relações Int., Brasília, v. 2, n.2, p. 199-219, jul./dez. 2004 